DOI: 10.17516/1997-1397-2021-14-5-647-658

УДК 517.55

\title{
Connecting Homomorphism and Separating Cycles
}

\author{
Roman V. Ulvert* \\ Siberian Federal University \\ Krasnoyarsk, Russian Federation \\ Reshetnev Siberian State University of Science and Technology \\ Krasnoyarsk, Russian Federation
}

Received 03.04.2021, received in revised form 11.06.2021, accepted 25.06.2021

\begin{abstract}
We discuss the construction of a long semi-exact Mayer-Vietoris sequence for the homology of any finite union of open subspaces. This sequence is used to obtain topological conditions of representation of the integral of a meromorphic $n$-form on an $n$-dimensional complex manifold in terms of Grothendieck residues. For such a representation of the integral to exist, it is necessary that the cycle of integration separates the set of polar hypersurfaces of the form. The separation condition in a number of cases turns out to be a sufficient condition for representing the integral as a sum of residues. Earlier, when describing such cases (in the works of Tsikh, Yuzhakov, Ulvert, etc.), the key was the condition that the manifold be Stein. The main result of this article is the relaxation of this condition.
\end{abstract}

Keywords: Mayer-Vietoris sequence, Grothendieck residue, separating cycle.

Citation: R.V. Ulvert, Connecting Homomorphism and Separating Cycles, J. Sib. Fed. Univ. Math. Phys., 2021, 14(5), 647-658. DOI: 10.17516/1997-1397-2021-14-5-647-658.

\section{Introduction}

In the theory of functions of one complex variable the Cauchy residue of a function $f$ at an isolated singular point $a$ is represented in a local coordinate $z$ by the integral over the cycle $\gamma^{(a)}=\{|z-a|=\varepsilon\}$ in a sufficiently small punctured neighborhood $U_{a} \backslash\{a\}$. The cycle $\gamma^{(a)}$ is called local cycle at $a$. By Cauchy's theorem the definition of the residue does not depend on the choice of a local cycle (choice of the local coordinate and the radius $\varepsilon$ ). It's usually not difficult to represent the integral

$$
\int_{\gamma} f d z
$$

of a meromorphic function $f$ over a cycle $\gamma$ lying outside the polar set of the function as a sum of residues: it suffices to know the homological expansion of the cycle $\gamma$ in terms of local cycles.

The multidimensional analogue of the Cauchy residue is the Grothendieck residue of a meromorphic differential $n$-forms $\omega$ given on an $n$-dimensional complex-analytic manifold. This residue in turn is represented by the integral over a local $n$-cycle in a neighborhood of an isolated intersection point of polar hypersurfaces of $\omega$. In this case, it is possible to show that in order for the integral of a meromorphic form to be represented in terms of residues, it is necessary that the cycle of integration in a certain sense separates the set of polar hypersurfaces of the form. The most complete results on the characterization of such separating cycles and their relationship to

*ulvertrom@yandex.ru

(C) Siberian Federal University. All rights reserved 
local cycles in Stein manifolds are presented by Tsikh and Yuzhakov (see [5, 9]) (these results have recently been complemented in $[6,7])$. In this article, we develop a method for studying separating cycles, which makes it possible to weaken the homological conditions for the manifold and a family of polar hypersurfaces of integrating form, abandoning the Stain property of the manifold.

Our main tool is a generalization of the well-known Mayer-Vietoris long exact sequence. All the necessary information about the homology of the union of open subspaces (the homology of the topological space with open cover $\mathfrak{U}$ ) is discussed in Section 1. The case of the union of more than two subspaces leads to the study of the double complex of a cover and the Mayer-Vietoris spectral sequence (see [3]).

In Section 2 we construct a connecting homomorphism that allows us to get the long Mayer-Vietoris sequence for any finite cover. This sequence is not exact in the general case, but it is semi-exact. In obtaining our results, ideas from Gleason's article [4] are essentially used. The notion of the resolution for a cycle associated with an open covering of the space (the $\mathfrak{U}$-resolution) in [4] is not standard, but it is very appropriate in our opinion.

The main results are presented in Section 3. Note that our results are formulated under the assumption that the $(2 n-1)$-dimensional homology of the manifold is trivial and that the intersection of the set of $n$ polar hypersurfaces of the integrating form $\omega$ is discrete. In terms of the corresponding long Mayer-Vietoris sequence, we have obtained (Theorem 3.1) a necessary and sufficient condition under which any separating cycle is represented in terms of local cycles (and therefore the integral is calculated in terms of the residues). However, this is only a reformulation of the problem in the language of homological algebra. Theorem 3.2 gives a more practical sufficient condition (in terms of homology of complements of polar hypersurfaces of the form $\omega$ ) under which any separating cycle is represented in terms of local cycles. This condition, in particular, is satisfied for Stein manifolds and arbitrary set of $n$ polar hypersurfaces, which allows us to obtain another proof of the Tsikh theorem on separating cycles in Stein manifolds (Theorem 3.3). Therefore, the condition from Theorem 3.2 gives the desired relaxation of the condition of Steinnes of the manifold.

\section{Homology of the union of open subspaces}

Let $\mathfrak{U}=\left\{U_{i}\right\}_{i \in I}$ be an open cover of topological space $X$, where $I$ is an ordered index set. We will use the standard notation $S_{q}(X)$ for the group of singular chains of dimension $q$ (with coefficients in $\mathbb{C}$ ) in $X$. Also, by $S_{*}=S_{*}(X)$ we will denote the corresponding chain complex with the boundary operator $\partial$.

Definition 1.1. A $\mathfrak{U}$-chain in $X$ of multiplicity $p$ and dimension $q$ is an alternating function $\sigma$ on $I^{p+1}$ with values

$$
\sigma\left(i_{0}, i_{1}, \ldots, i_{p}\right) \in S_{q}\left(U_{i_{0}} \cap U_{i_{1}} \cap \ldots \cap U_{i_{p}}\right),
$$

which vanishes except at a finite number of a points of $I^{p+1}$.

Note that $\mathfrak{U}$-chains can be identified with elements of the bigraded group

$$
C_{p, q}=\bigoplus_{i_{0}<i_{1}<\ldots<i_{p}} S_{q}\left(U_{i_{0}} \cap U_{i_{1}} \cap \ldots \cap U_{i_{p}}\right), \quad p, q=0,1, \ldots
$$

We denote by $S_{q}^{\mathfrak{U}}=S_{q}^{\mathfrak{U}}(X)$ the subgroup in $S_{q}=S_{q}(X)$ generated by singular $q$-simplices $\Delta$, such that $\operatorname{supp} \Delta \subset U_{i}$ for some $U_{i} \in \mathfrak{U}$. The natural inclusion $\iota: S_{*}^{\mathfrak{U}} \rightarrow S_{*}(X)$ obviously is a chain map. 
For the chain complex $C_{*}$ with the boundary operator $d$ we use the following standard notations for subgroups of cycles, subgroups of boundaries and homology groups:

$$
\begin{gathered}
Z_{q}\left(C_{*}\right)=\operatorname{ker}\left(d: C_{q} \rightarrow C_{q-1}\right), \\
B_{q}\left(C_{*}\right)=\operatorname{im}\left(d: C_{q+1} \rightarrow C_{q}\right), \\
H_{q}\left(C_{*}\right)=Z_{q}\left(C_{*}\right) / B_{q}\left(C_{*}\right) .
\end{gathered}
$$

The following fact shows that when calculating the homology of the space $X$ it is sufficient to use the complex $S_{*}^{\mathfrak{U}}$.

Theorem 1.1 (see [8]). The homomorphism $\iota_{*}: H\left(S_{*}^{\mathfrak{U}}\right) \rightarrow H(X)$ induced by the chain map $\iota: S_{*}^{\mathfrak{U}} \rightarrow S_{*}(X)$ is the isomorphism.

Consider first the case $I=\{1,2\}$. In this situation, there is the well-known long exact MayerVietoris sequence for homology of the union of two open subsets. This sequence is obtained from short exact sequences

$$
S_{q}^{\mathfrak{U}}(X) \stackrel{\varepsilon}{\longleftarrow} S_{q}\left(U_{1}\right) \oplus S_{q}\left(U_{2}\right) \stackrel{\delta}{\longleftarrow} S_{q}\left(U_{1} \cap U_{2}\right),
$$

where $\varepsilon:\left(\sigma_{1}, \sigma_{2}\right) \mapsto \sigma_{1}+\sigma_{2}$ is an epimorphism and $\delta: \sigma \mapsto(\sigma,-\sigma)$ is a monomorphism, at that $\varepsilon$ and $\delta$ are chain maps. Passing to homology, we get sequences

$$
H_{q}\left(S_{*}^{\mathfrak{U}}\right) \stackrel{\varepsilon_{*}}{\longleftarrow} H_{q}\left(U_{1}\right) \oplus H_{q}\left(U_{2}\right) \stackrel{\delta_{*}}{\longleftarrow} H_{q}\left(U_{1} \cap U_{2}\right),
$$

in which in general $\varepsilon_{*}$ is not an epimorphism and $\delta_{*}$ is not a monomorphism. The following properties hold: 1) $\left.\operatorname{im} \delta_{*}=\operatorname{ker} \varepsilon_{*} ; 2\right)$ the connecting homomorphism $\varphi: H_{q}\left(S_{*}^{\mathfrak{U}}\right) \rightarrow H_{q-1}\left(U_{1} \cap U_{2}\right)$ induced by the multivalued map $\delta^{-1} \partial \varepsilon^{-1}$ is correctly defined, at that $\operatorname{im} \varphi=\operatorname{ker} \delta_{*}$ and $\operatorname{ker} \varphi=$ $\operatorname{im} \varepsilon_{*}$. We get the required long exact sequence:

$$
\ldots \longleftarrow H_{q-1}\left(U_{1} \cap U_{2}\right) \stackrel{\varphi}{\longleftarrow} H_{q}\left(S_{*}^{\mathfrak{U}}\right) \stackrel{\varepsilon_{*}}{\longleftarrow} H_{q}\left(U_{1}\right) \oplus H_{q}\left(U_{2}\right) \stackrel{\delta_{*}}{\longleftarrow} H_{q}\left(U_{1} \cap U_{2}\right) \longleftarrow \ldots,
$$

in which groups $H_{q}\left(S_{*}^{\mathfrak{U}}\right)$ are replaced by isomorphic groups $H_{q}(X)$.

Let now $\operatorname{card}(I) \geqslant 2$. We will describe a sequence of chain groups generalizing the short exact sequence (1). Inclusions $U_{i_{0}} \cap U_{i_{1}} \cap \ldots \cap U_{i_{p}} \hookrightarrow U_{i_{0}} \cap U_{i_{1}} \cap \ldots\left[i_{k}\right] \ldots \cap U_{i_{p}}, k=0, \ldots, p$, induce the Čech boundary operator $\delta: C_{p, q} \rightarrow C_{p-1, q}$ defined by the formula "alternating sum":

$$
(\delta \sigma)\left(i_{0}, i_{1}, \ldots, i_{p-1}\right)=\sum_{i \in I} \sigma\left(i, i_{0}, \ldots, i_{p-1}\right) .
$$

In turn, the inclusions $U_{i} \subset X$ induce the operator $\varepsilon: C_{0, q} \rightarrow S_{q}^{\mathfrak{U}}$, which acts according to the same "alternating sum" formula as follows:

$$
\varepsilon \sigma=\sum_{i \in I} \sigma(i)
$$

at that $\varepsilon \delta=0$. We obtain the following Mayer-Vietoris sequence for the groups of singular chains of the union

$$
0 \longleftarrow S_{q}^{\mathfrak{U}} \stackrel{\varepsilon}{\longleftarrow} C_{0, q} \stackrel{\delta}{\longleftarrow} C_{1, q} \stackrel{\delta}{\longleftarrow} C_{2, q} \stackrel{\delta}{\longleftarrow} \ldots
$$

Theorem 1.2 (see [1]). The sequence (2) is exact for all $q=0,1, \ldots$ 
If $\operatorname{card}(I)=2$ then this sequence coincides with the sequence (1). In what follows, we will assume that $\operatorname{card}(I)>2$. Maps $\delta: C_{p, *} \rightarrow C_{p-1, *}$ and $\varepsilon: C_{0, *} \rightarrow S_{*}^{\mathfrak{U}}$ are, as above, chain maps of the corresponding complexes with the boundary operator $\partial$. In passing to homology, the exact sequence (2) goes over into the sequence

$$
0 \longleftarrow H_{q}\left(S_{*}^{\mathfrak{U}}\right) \stackrel{\varepsilon_{*}}{\longleftarrow} H_{q}\left(C_{0, *}\right) \stackrel{\delta_{*}}{\longleftarrow} H_{q}\left(C_{1, *}\right) \stackrel{\delta_{*}}{\longleftarrow} H_{q}\left(C_{2, *}\right) \stackrel{\delta_{*}}{\longleftarrow} \ldots
$$

about which, in general, we can say that it is only semi-exact. In this case, the measure of "inexactness" is the homology groups of the sequence (3), considered as a chain complex with a boundary operator $\delta_{*}$ (or $\varepsilon_{*}$ ).

More abstract point of view on the generalization of the Mayer-Vietoris sequence for homology of the union relates to consideration of two spectral sequences of the double complex $C=\left(C_{p, q} ; \delta, \partial\right)$ (see $\left.[2,3]\right)$. This double complex is a first quarter complex $\left(C_{p, q}=0\right.$ for $p<0$ or $q<0)$. By adding to $C$ a column $\left(S_{q}^{\mathfrak{U}} ; \partial\right)$ and a chain map $\varepsilon: C_{0, *} \rightarrow S_{*}^{\mathfrak{U}}$ we get the extended double complex

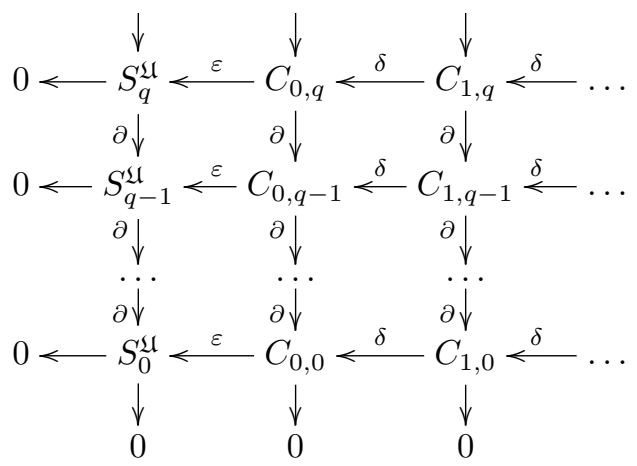

of singular chains which is dual to the familiar Čech-de Rham double complex for differential forms. Based on a double complex $C$ we build a total complex $T C$, formed by a graded group

$$
(T C)_{n}=\bigoplus_{p+q=n} C_{p, q}
$$

and a boundary operator $D:(T C)_{n} \rightarrow(T C)_{n-1}$ such that $\left.D\right|_{C_{p, q}}=\delta+(-1)^{p} \partial$.

The first of the spectral sequences $\left\{\left(E_{p, q}^{r} ; d^{r}\right)\right\}$ of the complex $C$ corresponds to filtration for $T C$ determined by the formula $F_{p}(T C)_{n}=\bigoplus_{i \leqslant p} C_{i, n-i}$. We have $E_{p, q}^{0}=C_{p, q}$ and $d^{0}= \pm \partial$, so $E_{p, q}^{1}=H_{q}\left(C_{p, *}\right)$ (the vertical homology of the complex $C$ ) and the differential $d^{1}: E_{p, q}^{1} \rightarrow E_{p-1, q}^{1}$ coincides with the map induced by the chain map $\delta: C_{p, *} \rightarrow C_{p-1, *}$, i.e. $d^{1}=\delta_{*}$. Further, the term $E_{p, q}^{2}$ (the horizontal homology of the vertical homology of the complex $C$ ) describes the homology of the sequence (3). Therefore, this spectral sequence (called the Mayer-Vietoris spectral sequence) is a generalization of the long exact Mayer-Vietoris sequence.

The second spectral sequence for $C$ is determined by another filtration of the total complex: $F_{p}(T C)_{n}=\bigoplus_{j \leqslant p} C_{n-j, j}$. In this case $E_{p, q}^{0}=C_{q, p}$, so $E^{1}$ is determined by the horizontal homology of the complex $C$. Since the strings of the extended complex (4) are exact, then $E_{p, 0}^{1}=S_{p}^{\mathfrak{U}}$ and $E_{p, q}^{1}=0$ for $q>0$. Considering the vertical homology, we obtain $E_{p, 0}^{2}=H_{p}(X)$ and $E_{p, q}^{2}=0$ for $q>0$. This means that the second spectral sequence "degenerates" and gives the isomorphism $H_{q}(T C) \cong H_{q}\left(S_{*}^{\mathfrak{U}}\right)$. Thus, the double complex $C$ "calculates" the homology of the space $X$. 


\section{Connecting homomorphism}

Pursuing the goal of finding a generalization of the long exact Mayer-Vietoris sequence in the case of any finite open cover of the space $X$, we construct a homomorphism, which is a connecting homomorphism for semi-exact sequences (3).

We will use the following notation:

$$
C_{p, q}^{\partial}=Z_{q}\left(C_{p, *}\right)=\operatorname{ker}\left(\partial: C_{p, q} \rightarrow C_{p, q-1}\right) .
$$

For the chain complex (2) consider the subcomplex

$$
0 \longleftarrow Z_{q}\left(S_{*}^{\mathfrak{U}}\right) \stackrel{\varepsilon}{\longleftarrow} C_{0, q}^{\partial} \stackrel{\delta}{\longleftarrow} C_{1, q}^{\partial} \stackrel{\delta}{\longleftarrow} C_{2, q}^{\partial} \stackrel{\delta}{\longleftarrow} \ldots
$$

In general, there are nontrivial homology groups $H_{p}\left(C_{*, q}^{\partial}\right)=Z_{p}\left(C_{*, q}^{\partial}\right) / B_{p}\left(C_{*, q}^{\partial}\right), p=0,1, \ldots$

Lemma 2.1. Let $\xi$ be the cycle belongs to $Z_{p}\left(C_{*, q}^{\partial}\right)$, where $p \geqslant 0, q \geqslant 1$, and $\xi=\delta \xi_{p+1}$ for some $\mathfrak{U}$-chain $\xi_{p+1} \in C_{p+1, q}$. Then $\partial \xi_{p+1}$ belongs to $Z_{p+1}\left(C_{*, q-1}^{\partial}\right)$ and the homology class $\left[\partial \xi_{p+1}\right] \in$ $H_{p+1}\left(C_{*, q-1}^{\partial}\right)$ depends only on the class $[\xi] \in H_{p}\left(C_{*, q}^{\partial}\right)$. The correspondence $[\xi] \rightarrow\left[\partial \xi_{p+1}\right]$ defines a homomorphism

$$
\varphi_{p+1}=\left(\partial \delta^{-1}\right)_{*}: H_{p}\left(C_{*, q}^{\partial}\right) \rightarrow H_{p+1}\left(C_{*, q-1}^{\partial}\right) .
$$

Proof. Note that the existence of a $\mathfrak{U}$-chain $\xi_{p+1}$ such that $\xi=\delta \xi_{p+1}$ follows from the fact that strings of the complex (4) are exact, due to condition $\delta \xi=0(\varepsilon \xi=0$ for $p=0)$. We have $\partial\left(\partial \xi_{p+1}\right)=0, \delta\left(\partial \xi_{p+1}\right)=\partial\left(\delta \xi_{p+1}\right)=\partial \xi=0($ see the diagram $(5))$, so $\partial \xi_{p+1} \in Z_{p+1}\left(C_{*, q-1}^{\partial}\right)$.
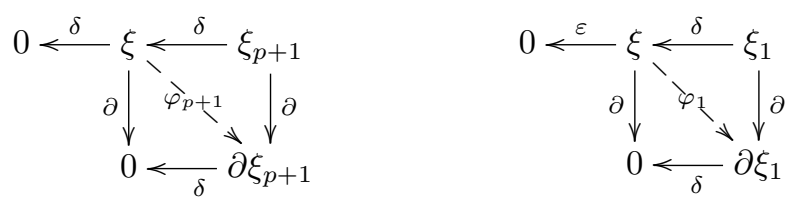

If also $\xi=\delta \xi_{p+1}^{\prime}$, then $\delta\left(\xi_{p+1}-\xi_{p+1}^{\prime}\right)=0$. So there exists a $\mathfrak{U}$-chain $\tau \in C_{p+2, q}$ such that $\xi_{p+1}-\xi_{p+1}^{\prime}=\delta \tau$. This implies $\xi_{p+1}^{\prime}=\xi_{p+1}-\delta \tau$. We have $\partial \xi_{p+1}^{\prime}=\partial\left(\xi_{p+1}-\delta \tau\right)=\partial \xi_{p+1}-\partial(\delta \tau)$, at that $\partial(\delta \tau) \in B_{p+1}\left(C_{*, q-1}^{\partial}\right)$, because $\partial(\delta \tau)=\delta(\partial \tau)$, where $\partial(\partial \tau)=0$. Hence it follows $\left[\partial \xi_{p+1}\right]=\left[\partial \xi_{p+1}^{\prime}\right] \in H_{p+1}\left(C_{*, q-1}^{\partial}\right)$.

Finally, let $\xi^{\prime}$ be an arbitrary representative of the class $[\xi] \in H_{p}\left(C_{*, q}^{\partial}\right)$. Then there exist a $\mathfrak{U}$ chain $\sigma \in C_{p+1, q}$, for which $\xi-\xi^{\prime}=\delta \sigma$ and $\partial \sigma=0$. If $\delta \xi_{p+1}=\xi$, then $\delta\left(\xi_{p+1}-\sigma\right)=\xi-\delta \sigma=\xi^{\prime}$. The class $\left[\xi^{\prime}\right]$ maps to the class of the $\mathfrak{U}$-chain $\partial\left(\xi_{p+1}-\sigma\right)=\partial \xi_{p+1}-\partial \sigma=\partial \xi_{p+1}$, which was required.

It remains to note that for the map $[\xi] \mapsto\left[\partial \xi_{p+1}\right]$ the image of the sum of classes is obviously equal to the sum of the images, so this map is a homomorphism.

Remark 2.1. We have $C_{p, 0}^{\partial}=C_{p, 0}$ for $p \geqslant 0$, so $H_{p}\left(C_{*, 0}^{\partial}\right)=H_{p}\left(C_{*, 0}\right)=0$. Hence for $q=1$ the homomorphism described in the Lemma 2.1 is trivial.

Lemma 2.2. Let be the cycle belongs to $\xi Z_{q}\left(S_{*}^{\mathfrak{U}}\right)$, where $q \geqslant 1$, and $\xi=\varepsilon \xi_{0}$ for some $\mathfrak{U}$-chain $\xi_{0} \in C_{0, q}$. Then $\partial \xi_{0}$ belongs to $Z_{0}\left(C_{*, q-1}^{\partial}\right)$ and the homology class $\left[\partial \xi_{0}\right] \in H_{0}\left(C_{*, q-1}^{\partial}\right)$ depends only on the class $[\xi] \in H_{q}\left(S_{*}^{\mathfrak{U}}\right)$. The correspondence $[\xi] \rightarrow\left[\partial \xi_{0}\right]$ defines a homomorphism

$$
\varphi_{0}=\left(\partial \varepsilon^{-1}\right)_{*}: H_{q}\left(S_{*}^{\mathfrak{U}}\right) \rightarrow H_{0}\left(C_{*, q-1}^{\partial}\right) .
$$


Proof. Existence of a $\mathfrak{U}$-chain $\xi_{0}$, such that $\xi=\varepsilon \xi_{0}$, follows from the surjectivity of $\varepsilon$. The fact that $\partial \xi_{0} \in Z_{0}\left(C_{*, q-1}^{\partial}\right)$ and independence of the class $\left[\partial \xi_{0}\right] \in H_{0}\left(C_{*, q-1}^{\partial}\right)$ from choice of $\xi_{0}$ can be proved in the same way as in Lemma 2.1 .

Let $\xi^{\prime}$ represents a class $[\xi] \in H_{q}\left(S_{*}^{\mathfrak{U}}\right)$. So there exists a chain $\sigma \in S_{q+1}^{\mathfrak{U}}$, such that $\xi-\xi^{\prime}=\partial \sigma$. Since $\varepsilon$ is an epimorphism, there exists $\tau \in C_{0, q+1}$, such that $\sigma=\varepsilon \tau$. We have $\varepsilon\left(\xi_{0}-\partial \tau\right)=\xi-\varepsilon(\partial \tau)=\xi-\partial(\varepsilon \tau)=\xi-\partial \sigma=\xi^{\prime}$, therefore $\partial$-cycle $\xi^{\prime}$ corresponds to the class $\left[\partial\left(\xi_{0}-\partial \tau\right)\right]=\left[\partial \xi_{0}-\partial \partial \tau\right]=\left[\partial \xi_{0}\right]$. In this way the map $[\xi] \mapsto\left[\partial \xi_{0}\right]$ is correctly defined. Obviously, this is a homomorphism.

Remark 2.2. For $q=1$ the homomorphism $\varphi_{0}$ is trivial.

The last two lemmas allow us to write the following "diagonal" sequence of maps:

$$
H_{r}\left(S_{*}^{\mathfrak{U}}\right) \stackrel{\varphi_{0}}{\longrightarrow} H_{0}\left(C_{*, r-1}^{\partial}\right) \stackrel{\varphi_{1}}{\longrightarrow} H_{1}\left(C_{*, r-2}^{\partial}\right) \rightarrow \ldots \rightarrow H_{r-2}\left(C_{*, 1}^{\partial}\right) \stackrel{\varphi_{r-1}}{\longrightarrow} H_{r-1}\left(C_{*, 0}^{\partial}\right) \cong 0 .
$$

The sequence of homomorphisms (6) is related to the following notion of a resolution for a cycle by Gleason (see [4]).

Definition 2.1. A $\mathfrak{U}$-resolution for the cycle $\xi \in Z_{r}\left(S_{*}^{\mathfrak{U}}\right)$ is a sequence $\left\{\xi_{p}\right\}_{p=0}^{r}$ of $\mathfrak{U}$-chains, $\xi_{p} \in C_{p, r-p}$, such that:

1) $\varepsilon \xi_{0}=\xi$

2) $\delta \xi_{p}=\partial \xi_{p-1}, p=1, \ldots, r$.

Remark 2.3. The existence of a $\mathfrak{U}$-resolution $\left\{\xi_{p}\right\}$ for any cycle $\xi \in Z_{r}\left(S_{*}^{\mathfrak{U}}\right)$ follows from the fact that the strings of the complex (4) are exact. Gleason's definition of a resolution suggests that $\xi \in Z_{r}\left(S_{*}\right)$. So the condition $\xi \in Z_{r}\left(S_{*}^{\mathfrak{U}}\right)$ is given as a criterion for the existence of the resolution.

Remark 2.4. The $\mathfrak{U}$-resolution for a cycle $\xi$ (accurate to sign \pm in front of its term) is, in fact, such $D$-cycle, which represents the image of the class $[\xi]$ under the isomorphism $H_{q}\left(S_{*}^{\mathfrak{U}}\right) \rightarrow$ $H_{q}(T C)$. It is a "zig-zag" of the double complex $C$.

Comparing the definitions of homomorphisms $\varphi_{p+1}$ and $\varphi_{0}$ from Lemmas 2.1, 2.2 with Definition 2.1, we obtain the following statement.

Proposition 2.1. Let $\left\{\xi_{p}\right\}$ be the $\mathfrak{U}$-resolution of a cycle $\xi \in Z_{r}\left(S_{*}^{\mathfrak{U}}\right)$. Then the sequence of images of the cycle $[\xi] \in H_{r}\left(S_{*}^{\mathfrak{U}}\right)$ under homomorphisms (6) has the form

$$
[\xi] \longmapsto\left[\partial \xi_{0}\right] \longmapsto\left[\partial \xi_{1}\right] \longmapsto \ldots \longmapsto\left[\partial \xi_{r-2}\right] \longmapsto\left[\partial \xi_{r-1}\right]=[0] .
$$

In a similar way, one can consider a part of the sequence of homomorphisms (6) starting from the group $H_{p}\left(C_{*, q}^{\partial}\right)$ for $p \geqslant 0$ and $q \geqslant 1$. In this case, we have the following sequence of homomorphisms

$$
H_{p}\left(C_{*, q}^{\partial}\right) \rightarrow H_{p+1}\left(C_{*, q-1}^{\partial}\right) \rightarrow \ldots \rightarrow H_{p+q-1}\left(C_{*, 1}^{\partial}\right) \rightarrow H_{p+q}\left(C_{*, 0}^{\partial}\right) \simeq 0,
$$

which is naturally leads to following version of the notion of the $\mathfrak{U}$-resolution.

Definition 2.2. Let $\xi$ by a $\mathfrak{U}$-chain of the multiplicity $p \geqslant 0$ and the dimension $q \geqslant 1$ such that $\partial \xi=0$ and $\delta \xi=0(\varepsilon \xi=0$ for $p=0)$. A sequence $\left\{\xi_{k}\right\}$ of $\mathfrak{U}$-chains $\xi_{k} \in C_{k, p+q-k+1}$, is said to be the $\mathfrak{U}$-resolution of the $\xi$, if the following conditions hold:

1) $\delta \xi_{p+1}=\xi$;

2) $\delta \xi_{k}=\partial \xi_{k-1}, k=p+2, \ldots, p+q+1$. 
Remark 2.5. As in the case $\xi \in Z_{r}\left(S_{*}^{\mathfrak{U}}\right)$, the resolution exists for any cycle $\xi \in Z_{p}\left(C_{*, q}^{\partial}\right)$.

The existence of the connecting homomorphism assumes that the open cover $\mathfrak{U}$ of topological space $X$ is finite $(\operatorname{card}(I)<\infty)$. In what follows, we will assume that this covering consists of $m(m \geqslant 2)$ elements.

In this case, for the double complex (4), we have $C_{p, q} \cong 0$ for $p \geqslant m$. Since the strings of the complex are exact, we see that $\delta: C_{m-1, *} \rightarrow C_{m-2, *}$ is monomorphism. Hence

$$
Z_{m-1}\left(C_{*, q}\right) \cong 0, \quad H_{m-1}\left(C_{*, q}^{\partial}\right)=H_{m-1}\left(C_{*, q}\right) \cong 0 .
$$

For any resolution $\left\{\xi_{k}\right\}$ of a cycle $\xi \in Z_{r}\left(S_{*}^{\mathfrak{U}}\right), r \geqslant m$, we have $\xi_{k}=0$ for all $k>m-1$, at that $\partial \xi_{m-1}=0$. We assume that the $\partial$-cycle $\xi_{m-1}$ is the end term of this resolution, ignoring the following zero terms. We will proceed similarly with the resolution of cycle $\xi \in Z_{p}\left(C_{*, q}^{\partial}\right)$.

The following statement is the last step to the construction of the desired generalization of a connecting homomorphism.

Lemma 2.3. Let $\xi \in Z_{m-3}\left(C_{*, q}^{\partial}\right), q \geqslant 1$, and let $\left\{\xi_{m-2}, \xi_{m-1}\right\}$ be the resolution of $\xi$. The correspondence of classes $[\xi] \in H_{m-3}\left(C_{*, q}^{\partial}\right)$ and $\left[\xi_{m-1}\right] \in H_{q-1}\left(C_{m-1, *}\right)$ defines correctly a homomorphism of homology groups

$$
\psi_{m-1}=\left(\delta^{-1} \partial \delta^{-1}\right)_{*}: H_{m-3}\left(C_{*, q}^{\partial}\right) \rightarrow H_{q-1}\left(C_{m-1, *}\right) .
$$

Proof. The action of the homomorphism $\psi_{m-1}$ is illustrated by the following diagram:

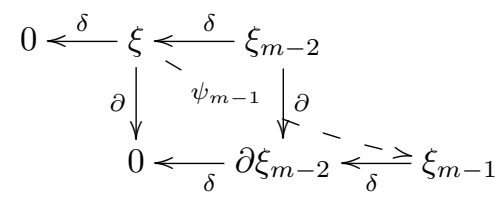

First, we show that the image $\left[\xi_{m-1}\right]$ does not depend on the choice of the resolution. Let $\left\{\xi_{m-2}, \xi_{m-1}\right\}$ and $\left\{\xi_{m-2}^{\prime}, \xi_{m-1}^{\prime}\right\}$ are resolutions of $\xi \in Z_{m-3}\left(C_{*, q}^{\partial}\right)$. We have $\delta\left(\xi_{m-2}-\xi_{m-2}^{\prime}\right)=$ $=\delta \xi_{m-2}-\delta \xi_{m-2}^{\prime}=\xi-\xi=0$, so there is (the only one) $\mathfrak{U}$-chain $\tau \in C_{m-1, q}$ for which $\delta \tau=\xi_{m-2}-\xi_{m-2}^{\prime}$. Hence $\xi_{m-2}^{\prime}=\xi_{m-2}-\delta \tau$, and

$$
\delta \xi_{m-1}^{\prime}=\partial \xi_{m-2}^{\prime}=\partial\left(\xi_{m-2}-\delta \tau\right)=\partial \xi_{m-2}-\partial(\delta \tau)=\delta \xi_{m-1}-\delta(\partial \tau)=\delta\left(\xi_{m-1}-\partial \tau\right) .
$$

Since $\delta: C_{m-1, *} \rightarrow C_{m-2, *}$ is a monomorphism, we get $\xi_{m-1}^{\prime}=\xi_{m-1}-\partial \tau$. Therefore $\left[\xi_{m-1}^{\prime}\right]=$ $=\left[\xi_{m-1}\right]$ in $H_{q-1}\left(C_{m-1, *}\right)$.

Next, we will show that the image $\left[\xi_{m-1}\right]$ also does not depend on the choice of the cycle representing the class in $H_{m-3}\left(C_{*, q}^{\partial}\right)$. If $[\zeta]=[\xi]$ then $\zeta=\xi+\delta \sigma$, where $\partial \sigma=0$. We put $\zeta_{m-2}=\xi_{m-2}+\sigma, \zeta_{m-1}=\xi_{m-1}$. We have $\delta \zeta_{m-2}=\delta\left(\xi_{m-2}+\sigma\right)=\delta \xi+\delta \sigma=\xi+\delta \sigma=\zeta$,

$$
\partial \zeta_{m-2}=\partial\left(\xi_{m-2}+\sigma\right)=\partial \xi_{m-2}+\partial \sigma=\partial \xi_{m-2}=\delta \xi_{m-1}=\delta \zeta_{m-1} .
$$

Hence, $\left\{\zeta_{m-2}, \zeta_{m-1}\right\}$ is the resolutions for $\zeta$, at that $\zeta_{m-1}=\xi_{m-1}$.

In order to prove that this correspondence of homology classes is a homomorphism, it suffices to note that a resolution for the sum of $\mathfrak{U}$-chains is the sum (term-by-term) of resolutions.

Remark 2.6. Let $m=2$. For the cycle $\xi \in Z_{r}\left(S_{*}^{\mathfrak{U}}\right)$ and its resolution $\left\{\xi_{0}, \xi_{1}\right\}$ similarly the correspondence $[\xi] \mapsto\left[\xi_{1}\right]$ gives the homomorphism

$$
\psi_{1}=\left(\delta^{-1} \partial \varepsilon^{-1}\right)_{*}: H_{r}\left(S_{*}^{\mathfrak{U}}\right) \rightarrow H_{r-1}\left(C_{1, *}\right),
$$

which is the connecting homomorphism $\varphi$ for the usual long exact Mayer-Vietoris sequence. 
Consider the homomorphisms $\varphi_{0}, \varphi_{1}, \ldots, \varphi_{m-3}$ from the sequence (6) and complement them with the homomorphism $\psi_{m-1}$ from Lemma 2.3. We obtain a sequence of homomorphisms whose action, according to Proposition 2.1 and Lemma 2.3, is described in terms of a resolution of the cycle as follows.

Theorem 2.1. For any $r \geqslant m$ there exists a sequence of homomorphisms

$$
H_{r}\left(S_{*}^{\mathfrak{U}}\right) \stackrel{\varphi_{0}}{\longrightarrow} H_{0}\left(C_{*, r-1}^{\partial}\right) \stackrel{\varphi_{1}}{\longrightarrow} \ldots \stackrel{\varphi_{m-3}}{\longrightarrow} H_{m-3}\left(C_{*, r-m+2}^{\partial}\right) \stackrel{\psi_{m-1}}{\longrightarrow} H_{r-m+1}\left(C_{m-1, *}\right),
$$

given by the following sequence of images:

$$
[\xi] \longmapsto\left[\partial \xi_{0}\right] \longmapsto \ldots \longmapsto\left[\partial \xi_{m-3}\right] \longmapsto\left[\xi_{m-1}\right],
$$

where $\left\{\xi_{p}\right\}$ is the resolution for the cycle $\xi \in Z_{r}\left(S_{*}^{\mathfrak{U}}\right)$.

Finally, taking the composition of all homomorphisms from (7), we obtain the desired connecting homomorphism $\varphi=\psi_{m-1} \varphi_{m-3} \ldots \varphi_{1} \varphi_{0}=\left(\delta^{-1} \partial \delta^{-1} \ldots \partial \delta^{-1} \partial \varepsilon^{-1}\right)_{*}$.

Theorem 2.2. Let $\mathfrak{U}=\left\{U_{i}\right\}$ be a finite open cover of a topological space $X$, consisting of $m \geqslant 2$ elements. Then the correspondence of homology classes $[\xi] \rightarrow\left[\xi_{m-1}\right]$, where $\xi \in Z_{r}\left(S_{*}^{\mathfrak{U}}\right)$ and $\left\{\xi_{p}\right\}$ is arbitrary $\mathfrak{U}$-resolution of cycle $\xi$, defines a connecting homomorphism

$$
\varphi: H_{r}\left(S_{*}^{\mathfrak{U}}\right) \rightarrow H_{r-m+1}\left(C_{m-1, *}\right) .
$$

For $m>2$ this homomorphism generates a semi-exact long sequence of homology groups

$$
\begin{aligned}
& \ldots \longleftarrow H_{q-m+1}\left(C_{m-2, *}\right) \stackrel{\delta_{*}}{\longleftarrow} H_{q-m+1}\left(C_{m-1, *}\right) \stackrel{\varphi}{\longleftarrow} H_{q}\left(S_{*}^{\mathfrak{U}}\right) \stackrel{\varepsilon_{*}}{\longleftarrow} H_{q}\left(C_{0, *}\right) \stackrel{\delta_{*}}{\longleftarrow} \ldots \\
& \ldots \stackrel{\delta_{*}}{\longleftarrow} H_{q}\left(C_{m-1, *}\right) \stackrel{\varphi}{\longleftarrow} H_{q+m-1}\left(S_{*}^{\mathfrak{U}}\right) \stackrel{\varepsilon_{*}}{\longleftarrow} H_{q+m-1}\left(C_{0, *}\right) \longleftarrow \ldots
\end{aligned}
$$

Proof. It remains to show that $\operatorname{im} \varphi \subset \operatorname{ker} \delta_{*}$ and $\operatorname{im} \varepsilon_{*} \subset \operatorname{ker} \varphi$. The first inclusion follows from the equality $\delta_{*}\left[\xi_{m-1}\right]=\left[\delta \xi_{m-1}\right]=\left[\partial \xi_{m-2}\right]=0$. Let us prove the second inclusion. Let $[\xi] \in \operatorname{im} \varepsilon_{*}$. Then $\xi=\varepsilon \xi_{0}$ for some $\mathfrak{U}$-chain $\xi_{0} \in C_{0, q}$, and $\partial \xi_{0}=0$. Therefore $\varphi_{0}[\xi]=\left[\partial \xi_{0}\right]=0$, and hence $\varphi[\xi]=0$.

Remark 2.7. In what follows, we will assume that the codomain of the connecting homomorphism is the subgroup $H_{q-m+1}^{\text {sep }}\left(C_{m-1, *}\right)=\operatorname{ker} \delta_{*} \subset H_{q-m+1}\left(C_{m-1, *}\right)$. The notation $H_{q}^{\mathrm{sep}}\left(C_{m-1, *}\right)$ will be discussed later. In this way,

$$
\varphi: H_{q}\left(S_{*}^{\mathfrak{U}}\right) \rightarrow H_{q-m+1}^{\mathrm{sep}}\left(C_{m-1, *}\right) .
$$

Similarly, from the previous proof, we can conclude that $\operatorname{im} \psi_{m-1} \subset \operatorname{ker} \delta_{*}$. Therefore, we further assume that $\psi_{m-1}: H_{m-3}\left(C_{*, q}^{\partial}\right) \rightarrow H_{q-1}^{\mathrm{sep}}\left(C_{m-1, *}\right)$.

The proofs of the following properties of the homomorphisms $\varphi_{0}, \varphi_{p+1}$ and $\psi_{m-1}$, from which the connecting homomorphism is "glued", are completely standard. Moreover, conditions on the homology appear as sufficient conditions for inverting the required vertical arrows of the complex (4) at diagrammatic search.

Lemma 2.4. If $H_{q-1}\left(C_{0, *}\right) \cong 0$, then the homomorphism $\varphi_{0}: H_{q}\left(S_{*}^{\mathfrak{U}}\right) \rightarrow H_{0}\left(C_{*, q-1}^{\partial}\right)$ is an epimorphism. If $H_{q}\left(C_{0, *}\right) \cong H_{q-1}\left(C_{1, *}\right) \cong 0$, then it is a monomorphism.

Lemma 2.5. If $H_{q-1}\left(C_{p+1, *}\right) \cong 0$, then the homomorphism $\varphi_{p+1}: H_{p}\left(C_{*, q}^{\partial}\right) \rightarrow H_{p+1}\left(C_{*, q-1}^{\partial}\right)$ is an epimorphism. If $H_{q-1}\left(C_{p+2, *}\right) \cong 0$, then it is a monomorphism. 
Lemma 2.6. The homomorphism $\psi_{m-1}: H_{m-3}\left(C_{*, q}^{\partial}\right) \rightarrow H_{q-1}^{s e p}\left(C_{m-1, *}\right)$ is an isomorphism.

Remark 2.8. For $m=2$ the homomorphism $\psi_{1}: H_{q}\left(S_{*}^{\mathfrak{U}}\right) \rightarrow H_{q-1}^{\text {sep }}\left(C_{1, *}\right)$ is only an epimorphism in general case.

Considering that the composition of epimorphisms is an epimorphism, and the composition of monomorphisms is a monomorphism, we obtain the following property of the connecting homomorphism.

Theorem 2.3. For the connecting homomorphism

$$
\varphi: H_{q}\left(S_{*}^{\mathfrak{U}}\right) \rightarrow H_{q-m+1}^{s e p}\left(C_{m-1, *}\right)
$$

to be an epimorphism, it suffices to satisfy the condition

$$
H_{q-1}\left(C_{0, *}\right) \cong H_{q-2}\left(C_{1, *}\right) \cong \ldots \cong H_{q-m+2}\left(C_{m-3, *}\right) \cong 0,
$$

and for $\varphi$ to be a monomorphism, it suffices to satisfy the condition

$$
H_{q}\left(C_{0, *}\right) \cong H_{q-1}\left(C_{1, *}\right) \cong \ldots \cong H_{q-m+2}\left(C_{m-2, *}\right) \cong 0 .
$$

Remark 2.9. The homomorphism $\varphi: H_{q}\left(S_{*}^{\mathfrak{U}}\right) \rightarrow H_{q-m+1}^{\text {sep }}\left(C_{m-1, *}\right)$ is surjective if and only if the sequence (8) is exact in the term $H_{q-m+1}\left(C_{m-1, *}\right)$.

Remark 2.10. The condition (9) can be replaced by the following weaker condition: if $\xi \in$ $C_{p, q-p-1}$ such that $\partial \xi=0$ and $\delta \xi=0(\varepsilon \xi=0)$, then $[\xi]=0$ in $H_{q-p-1}\left(C_{p, *}\right), p=0, \ldots, m-3$.

\section{Separating cycles}

The notion of the separating cycle appeared in complex analysis in connection with a property of the Grothendieck residue. Let $\omega$ be a meromorphic $n$-form on an $n$-dimensional complexanalytic manifold $M$, and $F_{1}, \ldots, F_{n}$ are polar hypersurfaces of $\omega, F=F_{1} \cup \ldots \cup F_{n}$. In a sufficiently small neighborhood $U_{a}$ of an isolated point $a$ of the intersection $Z=F_{1} \cap \ldots \cap F_{n}$ the form $\omega$ is given by

$$
\omega=\frac{h(z) d z_{1} \wedge \ldots \wedge d z_{n}}{f_{1}(z) \ldots f_{n}(z)}
$$

where $h, f_{1}, \ldots f_{n}$ are holomorphic germs at $a,\left.F_{k}\right|_{U_{a}}=\left\{f_{k}=0\right\}$. The grothendieck residue of the form $\omega$ at the point $a$ is represented by the integral

$$
\operatorname{res}_{a} \omega=\frac{1}{(2 \pi i)^{n}} \int_{\gamma^{(a)}} \omega,
$$

where $\gamma^{(a)}$ is a local cycle at $a$ having the form

$$
\gamma^{(a)}=\left\{z \in U_{a}:\left|f_{1}(z)\right|=\varepsilon_{1}, \ldots,\left|f_{n}(z)\right|=\varepsilon_{n}\right\} .
$$

The orientation of $\gamma^{(a)}$ is determined by the condition $d\left(\arg f_{1}\right) \wedge \ldots \wedge d\left(\arg f_{n}\right) \geqslant 0$. It is not hard to see that $\gamma^{(a)} \in Z_{n}(M \backslash F)$.

The mentioned property of the residue (12) is as follows: it is zero if $h$ belongs to the ideal generated by $f_{1}, \ldots, f_{n}$ in the ring $\mathcal{O}_{a}$ of germs of holomorphic functions. This property is of a topological nature. Indeed, if $h=h_{j} f_{j}$, then $\omega$ has poles only on the $n-1$ hypersurfaces $F_{k}=\left\{f_{k}=0\right\}, k=1, \ldots[j] \ldots, n$, in the complement of which the $n$-cycle $\gamma^{(a)}$ becomes homologically trivial. Indeed, $\gamma^{(a)}$ is a boundary of the $(n+1)$-chain $\sigma_{j}=\left\{\left|f_{1}\right|=\varepsilon_{1}, \ldots,\left|f_{j}\right| \leqslant\right.$ $\left.\varepsilon_{j}, \ldots,\left|f_{n}\right|=\varepsilon_{n}\right\}$ taken with a suitable orientation, so $\gamma^{(a)} \sim 0$. Therefore, according to Stokes' formula, the integral (12) for the $h=h_{j} f_{j}$ is zero. 
Definition 3.1. A n-dimensional cycle $\Gamma \in Z_{n}(M \backslash F)$ separates hypersurfaces $F_{1}, \ldots, F_{n}$, if it satisfies the conditions

$$
\Gamma \sim 0 \text { in } M \backslash\left(F_{1} \cup \ldots[j] \ldots \cup F_{n}\right) \text { for all } j=1, \ldots, n \text {. }
$$

By the above, the local cycle $\gamma^{(a)}$ from the definition of the Grothendieck residue separates the set of polar hypersurfaces of $\omega$.

An important argument for the use of Grothendieck residues of meromorphic forms is their rational computability in terms of a finite number of Taylor coefficients of functions $h, f_{1}, \ldots, f_{n}$ at the point $a$. In this connection there is a problem of the representation of the integral of a meromorphic form $\omega$ by residues. The topological formulation of this problem is as follows. Let $\mathcal{F}=\left\{F_{1}, \ldots, F_{n}\right\}$ be a set of hypersurfaces in an $n$-dimensional complex-analytic manifold $M$. Let us denote by $F$ the union of these hypersurfaces, and by $Z_{0}$ the discrete part of their intersection $Z=F_{1} \cap \ldots \cap F_{n}$. It is required to find out in which case the given $n$-cycle $\Gamma$ in $M \backslash F$ is homologically expressed in terms of local cycles $\gamma^{(a)}, a \in Z_{0}$. In view of the above, for this it is necessary the cycle $\Gamma$ separates the given set of hypersurfaces $\mathcal{F}$.

We denote by $H_{n}^{\text {loc }}(M \backslash F)$ the subgroup in $H_{n}(M \backslash F)$ generated by the classes of all local cycles $\gamma^{(a)}, a \in Z_{0}$. We also denote by $H_{n}^{\operatorname{sep}}(M \backslash F)$ the subgroup of classes of all cycles separating the set of hypersurfaces $\mathcal{F}$. We have

$$
H_{n}^{\text {loc }}(M \backslash F) \subset H_{n}^{\mathrm{sep}}(M \backslash F) .
$$

We are interested in sufficient conditions on the manifold $M$ and the collection of hypersurfaces $\mathcal{F}$ under which $H_{n}^{\text {sep }}(M \backslash F)=H_{n}^{\text {loc }}(M \backslash F)$, that is, in which any separating cycle is homologically represented in terms of local cycles.

Consider the space $X=M \backslash Z$ and its cover $\mathfrak{U}$ formed by open sets $U_{j}=M \backslash F_{j}, j=1, \ldots, n$. We get the corresponding extended double complex (4) and the semi-exact sequence (8) for $q=m=n$. Given the isomorphism of Theorem 1.1, this sequence can be written as

$$
\cdots \longleftarrow H_{n}\left(C_{m-2, *}\right) \stackrel{\delta_{*}}{\longleftarrow} H_{n}(M \backslash F) \stackrel{\varphi}{\longleftarrow} H_{2 n-1}(M \backslash Z) \stackrel{\varepsilon_{*}}{\longleftarrow} H_{2 n-1}\left(C_{0, *}\right) \longleftarrow \ldots
$$

The condition for separating the set $\mathcal{F}$ by a cycle $\Gamma$ means that $\delta \Gamma$ is an $\partial$-boundary in the group $C_{n-2, n}$. So $H_{n}^{\text {sep }}(M \backslash F)=\operatorname{ker} \delta_{*}$, which explains the previously used (see Remark 2.7) notation for the subgroup $H_{q-m+1}^{\text {sep }}\left(C_{m-1, *}\right)=\operatorname{ker} \delta_{*} \subset H_{q-m+1}\left(C_{m-1, *}\right)$. Let us show that $H_{n}^{\text {loc }}(M \backslash F) \subset \operatorname{im} \varphi$.

It suffices to show that each generator $\left[\gamma^{(a)}\right], a \in Z_{0}$, of the group $H_{n}^{\text {loc }}(M \backslash F)$ have preimage in $H_{2 n-1}(M \backslash Z)$. For a fixed point $a \in Z_{0}$, consider the $(2 n-1)$-dimensional sphere $S_{a}$ centred at the point $a$ of a small radius. The class $\left[S_{a}\right]$ can be represented as a cycle $\partial \Pi_{a}$, where

$$
\Pi_{a}=\left\{z \in U_{a}:\left|f_{i}(z)\right|<\varepsilon_{i}, i=1, \ldots, n\right\},
$$

where the orientation of the special analytical polyhedron $\Pi_{a}$ is induced by the orientation of the manifold $M$. Moreover, the boundary $\partial \Pi_{a}$ of the polyhedron $\Pi_{a}$ is the sum of its $(n-1)$ dimensional faces $\tau_{j}=\left\{\left|f_{1}\right| \leqslant \varepsilon_{1}, \ldots,\left|f_{j}\right|=\varepsilon_{j}, \ldots,\left|f_{n}\right| \leqslant \varepsilon_{n}\right\}, j=1, \ldots, n$, taken with suitable orientation, at that $\operatorname{supp} \tau_{j} \subset U_{j}$. Therefore, $\partial \Pi_{a} \in Z_{2 n-1}\left(S_{*}^{\mathfrak{U}}\right)$, and for the cycle $\partial \Pi_{a}$ can be built the $\mathfrak{U}$-resolution $\left\{\xi_{p}\right\}$. It is directly verified that terms of the resolution can be taken as follows:

$$
\xi_{p}\left(i_{0}, i_{1}, \ldots, i_{p}\right)= \pm \tau_{i_{0}} \cap \tau_{i_{1}} \cap \ldots \cap \tau_{i_{p}} .
$$


Moreover, the final term $\xi_{n-1}=\xi_{n-1}(1, \ldots, n)$ of the resolution is the local cycle $\gamma^{(a)}$. So $\varphi\left[S_{a}\right]=\varphi\left[\Pi_{a}\right]=\left[\gamma^{(a)}\right]$, as required to prove. It also follows from the last reasoning that if the group $H_{2 n-1}(M \backslash Z)$ is generated by the classes of cycles $S_{a}, a \in Z_{0}$, in particular if $H_{2 n-1}(M) \cong 0$ and $Z=Z_{0}$, then $H_{n}^{\text {loc }}(M \backslash F)=\operatorname{im} \varphi$. This fact, with considering Remark 2.10, proves the following theorem.

Theorem 3.1. Let $H_{2 n-1}(M) \cong 0$ and let the intersection $Z=F_{1} \cap \ldots \cap F_{n}$ be discrete. Then the groups $H_{n}^{\text {sep }}(M \backslash F)$ and $H_{n}^{\text {loc }}(M \backslash F)$ are coincide if and only if the semi-exact sequence (14) is exact in the term $H_{n}(M \backslash F)$.

Remark 3.1. For $n=2$ the sequence (14) turns into a long exact Mayer-Vietoris sequence. Therefore, under the assumptions made on the manifold and the set of hypersurfaces, the equality $H_{2}^{\text {loc }}(M \backslash F)=H_{2}^{\text {sep }}(M \backslash F)$ is always the case.

Consider (see Remark 2.7) the connecting homomorphism $\varphi$ from the sequence (14) as the homomorphism

$$
\varphi: H_{2 n-1}(M \backslash Z) \rightarrow H_{n}^{\mathrm{sep}}(M \backslash F) .
$$

Sequence (14) is exact in the term $H_{n}(M \backslash F)$ if and only if $\varphi$ is an epimorphism (see Remark 2.9). Considering Theorem 2.3, we obtain the following sufficient condition.

Theorem 3.2. Let $H_{2 n-1}(M) \cong 0$ and let the intersection $Z=F_{1} \cap \ldots \cap F_{n}$ be discrete. Then for the equality of groups $H_{n}^{\text {sep }}(M \backslash F)=H_{n}^{\text {loc }}(M \backslash F)$ it suffices to satisfy the condition

$$
H_{2 n-2}\left(C_{0, *}\right) \cong H_{2 n-3}\left(C_{1, *}\right) \cong \ldots \cong H_{n+1}\left(C_{n-3, *}\right) \cong 0 .
$$

Remark 3.2. In last two theorems, instead the triviality of the group $H_{2 n-1}(M)$ and the discreteness of the intersection $Z=F_{1} \cap \ldots \cap F_{n}$, we can assume the following weaker condition: the group $H_{2 n-1}(M \backslash Z)$ is generated by classes of cycles $S_{a}, a \in Z_{0}$. The condition (16) can also be replaced (see Remark 2.10) by the following weaker condition: if $\xi \in C_{p, 2 n-p-2}$ such that $\partial \xi=0$ and $\delta \xi=0(\varepsilon \xi=0)$, then $[\xi]=0$ in group $H_{2 n-p-2}\left(C_{p, *}\right), p=0, \ldots, n-3$.

As a consequence of Theorem 3.2, it is easy to obtain the following theorem on separating cycles in Stein manifolds which was proved by Tsikh.

Theorem 3.3 (see [5]). Let $M$ be a Stein manifold of dimension $n$. Then the equality of groups $H_{n}^{\text {sep }}(M \backslash F)=H_{n}^{\text {loc }}(M \backslash F)$ holds for any set $\mathcal{F}=\left\{F_{1}, \ldots, F_{n}\right\}$ of hypersurfaces in $M$.

Proof. As it was noted in [5], it suffices to prove the statement of the theorem under the following assumptions: 1) $\left.H_{2 n-1}(M) \cong 0 ; 2\right) M \backslash F_{j}, j=1, \ldots, n$, are the Stein manifolds; 3) the intersection $Z=F_{1} \cap \ldots \cap F_{n}$ is discrete. It remains to note that all possible intersections of the sets $U_{j}=M \backslash F_{j}$ are also Stein manifolds. Condition (16) follows from the fact that for an arbitrary Stein manifold $X$ the homology groups (with coefficients in the field) $H_{q}(X)$ are trivial for $q>\operatorname{dim} X$.

Remark 3.3. In the case of the Stein manifold $M$ and an arbitrary set of hypersurfaces $\mathcal{F}$ in $M$ the connecting homomorphism $\varphi: H_{2 n-1}(M \backslash Z) \rightarrow H_{n}^{\operatorname{sep}}(M \backslash F)$ is an isomorphism. The injectivity follows from the fulfillment of conditions of the form (10).

This work is supported by the Krasnoyarsk Mathematical Center and financed by the Ministry of Science and Higher Education of the Russian Federation in the framework of the establishment and development of regional Centers for Mathematics Research and Education (Agreement No. 075-02-2021-1388). 


\title{
References
}

[1] R.Bott, L.W.Tu, Differential Forms in Algebraic Topology, New York, Springer-Verlag, 1982.

[2] K.S.Brown, Cohomology of groups, Graduate Texts in Mathematics, vol. 87, New York, Springer-Verlag, 1994, corrected reprint of the 1982 original.

[3] J.Chen, Z.Lü, J.Wu, Orbit configuration spaces of small covers and quasi-toric manifolds, Sci. China Math. 64(2021), 167-196. DOI: 10.1007/s11425-018-9526-6

[4] A.M.Gleason, The Cauchy - Weil theorem, J. Math. Mech., 12(1963), no. 3, 429-444.

[5] A.K.Tsikh, Multidimensional Residues and Their Applications, Providence, AMS, 1992.

[6] R.V.Ulvert, Homological Resolutions in Problems About Separating Cycles, Sib. Math. J., 59(2018), no. 3, 542-550. DOI: 10.1134/S0037446618030163

[7] R.V.Ulvert, On computability of multiple integrals by means of a sum of local residues, Sib. Èlektron. Mat. Izv. 15(2018), 996-1010 (Russian). DOI: 10.17377/semi.2018.15.084

[8] J.W.Vick, Homology Theory: An Introduction to Algebraic Topology, Graduate Texts in Mathematics, vol. 145, New York, Springer-Verlag, 2nd ed., 1994.

[9] A.P.Yuzhakov, The separating subgroup and local residues, Sib. Math. J., 29(1988), no. 6, 1028-1033 (Russian).

\section{Связывающий гомоморфизм и разделяющие циклы}

\author{
Роман В. Ульверт \\ Сибирский федеральный университет \\ Красноярск, Российская Федерация \\ Сибирский государственный университет науки и технологий им. М. Ф. Решетнева \\ Красноярск, Российская Федерация
}

\begin{abstract}
Аннотация. Обсуждается построение длинной полуточной последовательности МайераВиеториса для гомологий объединения конечного числа открытых подпространств. Эта последовательность применяется для получения топологических условий, при которых интеграл от мероморфной дифференциальной формы в многомерном комплексном многообразии представляется в виде суммы вычетов Гротендика. Для существования такого представления интеграла необходимо, чтобы цикл интегрирования разделял семейство полярных гиперповерхностей формы. Условие разделения в ряде случаев оказывается достаточным условием для представления интеграла в виде суммы вычетов. Ранее при описании таких случаев (в работах А. К. Циха, А. П. Южакова, Р. В. Ульверта и др.) ключевым оказывалось условие штейновости многообразия. Основным результатом данной статьи является ослабление этого условия.
\end{abstract}

Ключевые слова: последовательность Майера-Виеториса, вычет Гротендика, разделяющий цикл. 